\title{
REVIEWS
}

Adv Clin Exp Med 2014, 23, 4, 639-644

๑ Copyright by Wroclaw Medical University ISSN 1899-5276

Andrzej Gryglewski ${ }^{\text {A, B, E, F, Iwona Z. Pena }}{ }^{\text {B-D, F, Krzysztof A. Tomaszewski }}{ }^{\text {B-D, F, }}$, JERZY A. WALOCHA ${ }^{\text {A, E, F }}$

\section{Unsolved Questions Regarding the Role of Esophageal Hiatus Anatomy in the Development of Esophageal Hiatal Hernias}

\author{
Department of Anatomy, Jagiellonian University Medical College, Kraków, Poland
}

A - research concept and design; B - collection and/or assembly of data; C - data analysis and interpretation;

$\mathbf{D}$ - writing the article; $\mathbf{E}$ - critical revision of the article; $\mathbf{F}$ - final approval of article; $\mathbf{G}$ - other

\begin{abstract}
The association of esophageal hiatal hernias with gastro-esophageal reflux disease was recognized long ago, however its origins are still disputed. The anatomy of the diaphragm and esophagogastric junction appears to be crucial to hiatal hernia development. The aim of this paper was to perform a literature review to present the current state of knowledge regarding the anatomy and pathology of esophageal sliding hiatal hernias. An electronic journal search was undertaken to identify all relevant studies published in English regarding esophageal hiatal hernias. This search included the Medline and Embase databases from 1962 to 2013. The following keywords were used in various combinations: hiatus hernia, hiatal, gastro-esophageal reflux disease, etiology, anatomy, and esophageal. The nature of a hiatal hernia is complicated by the multifactorial etiology of the disease, which involves the interplay of genetic and environmental factors. Its anatomy is still a matter of dispute. The exact point at which hernia development begins has yet to be understood (Adv Clin Exp Med 2014, 23, 4, 639-644).
\end{abstract}

Key words: diaphragm, GERD, hiatus hernia.

The incidence of hiatal hernia has significantly increased over recent years [1]. Many patients suffering from such hernias seek therapeutic intervention to alleviate the gastro-intestinal symptoms caused primarily by acid reflux [2]. Standard diagnostic methods such as barium swallow contrast radiographs and endoscopy along with standard medical treatment, including surgical intervention, are already defined and generally accepted [3]. Although the direct cause of diaphragmatic hernia formation remains disputed, it appears that the etiology lies primarily in the anatomy of the diaphragm and the esophagogastric junction. Even though this region has recently been investigated by many authors, some elements of its construction still remain a matter of dispute [3-7].

The purpose of this paper was to perform a literature review to demonstrate the current state of knowledge regarding the anatomy and pathology of esophageal sliding hiatal hernias. Emphasis was placed on identifying whether hiatal hernia is a generalized or localized disease and whether or not a hiatal hernia with gastric fold protrusion should be considered a variation of a sliding hiatal hernia.

\section{Methods}

An electronic journal search was conducted to identify all studies published in English regarding esophageal hiatal hernias from 1962 to 2013, using the Medline and Embase databases. The following keywords were used in various combinations: hiatus hernia, hiatal, gastro-esophageal reflux disease, etiology, anatomy, and esophageal. The reference lists of all of the relevant studies and existing reviews were screened for additional relevant publications.

Each publication was initially assessed for relevance using the data presented in the abstract. When 
an abstract was not available or failed to provide sufficient information, a reprint of the full paper was obtained. When papers or abstracts reported different stages of clinical trials, only the longer-term studies were included in the review. When both a full paper and an abstract were published based on data from the same clinical trial, only the full paper was included. Finally, from the body of gathered literature, based on our clinical and research experience, the most relevant articles were chosen to form the knowledge base for this review paper.

\section{Results}

\section{The Anatomy of the Diaphragm}

From an anatomical point of view, the diaphragm is a tendinous, muscular, dome-shaped partition that separates the chest from the abdominal cavity (Fig. 1A). Muscle bundles radiate to their peripheral attachments from the clearly distinguished broad tendinous center of the diaphragm. From the sternal aspect, the diaphragm connects to the xiphoid process, to the cartilages of the seventh and eight ribs, to the cartilage and bone of the ninth rib, and to the bone portion of the tenth through twelfth ribs. The lumbar portion of the diaphragm consists of the anterior longitudinal ligament, which gives rise to the left and right crura, as well as the bilateral medial and lateral arcuate ligaments. The lateral arcuate ligament connects laterally to the twelfth rib and medially to the transverse process of the first lumbar vertebra. It forms the lateral lumbocostal arch which spans the quadratus lumborum muscle. From its medial attachment, the medial arcuate ligament spans the psoas muscle and attaches to the body of the first or second lumbar vertebra creating the medial lumbocostal arch.
Most commonly, the esophageal hiatus is formed by interlacing elements of the right diaphragmatic crus $[4,8]$. The right crus divides into a dorsal bundle, which forms the left limb of the structure, and a ventral bundle, which forms the right limb. As the muscle bundles approach the hiatus, the limbs cross each other in a scissor like fashion; the left limb passes to the upper left of the esophagus, while the right limb passes to the upper right [8]. The lateral fibers of each limb insert into the central tendon while the medial fibers form the hiatal margins and decussate at the midline in front of the esophagus [8]. In such a manner, the limbs of the right crus envelop the esophagus and form the hiatus (Fig. 1B). However, there are several anatomic variations in the formation of the esophageal hiatus, most notably in the relative contributions of muscle fibers from the right and left diaphragmatic crura. Loukas et al. [4] distinguished six different types of diaphragmatic muscular variants within the esophageal hiatal region. The most common variant, found in $45 \%$ of cadavers, consisted of the hiatus being formed by muscular bundles of the right crus alone as described above [4]. The other five variants involved varying degrees of contributions from the left crus [4]. Furthermore, after performing both cadaveric observations and a series of electrophysiological experiments involving stimulation of the diaphragm branches in patients scheduled for laparotomy, Shafik et al. [9] propose that there is a separate loop muscle surrounding the lower esophagus in the esophagogastric region. This muscle appears to function independently and is not derived from the diaphragmatic crura [9]. The exact anatomy of the diaphragmatic region and what causes observed muscular and hiatal differences within the area is still largely unknown. Further research is necessary to better define the anatomy and causes of its variation.
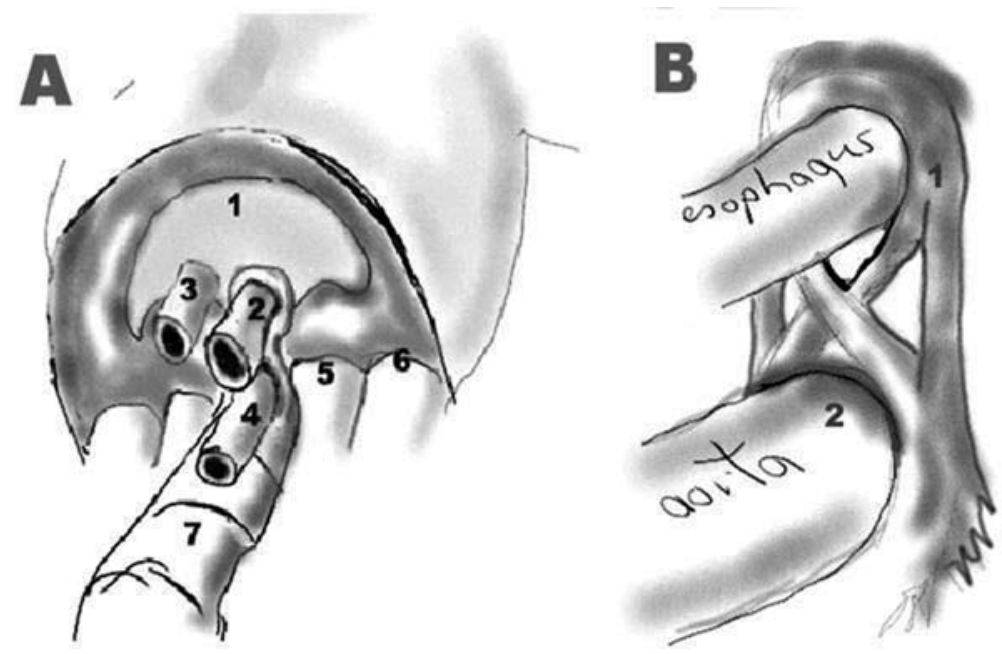

Fig. 1. A. Anatomy of diaphragm:

1 - Central tendon; 2 - Esophagus;

3 - Interior vena cava; 4 - Aorta;

5 - Medial arcuate ligament;

6 - Lateral arcuate ligament,

7 - Spine.

B. Hypothetical anatomy of the esophageal and aortic hiatus: 1 - Left crus creating the medial and lateral bundle; 2 - Medial arcuate ligament. (Fig. drawn by Andrzej Gryglewski) 


\section{Hiatal Hernia Types}

The proportion of the general population that is affected by hiatal hernias ranges from $10 \%$ to $50 \%$ [10]. Currently there are three distinguishable types of hiatal hernias: the sliding hernia, the paraesophageal hernia and a combination of the two $[3,11]$. Some authors consider the giant paraesophageal hernia as a fourth distinguishable type of hiatal hernia [12]. Each type of hernia presents with different symptoms and complications. Among the different types of hernias, the sliding hernia is the most problematic because its pathogenesis is not yet fully understood.

\section{The Pathogenesis of Sliding Hernias}

The association of hiatal hernias with gastroesophageal reflux disease (GERD) has long been recognized and is linked with the failure of antireflux mechanisms [13]. It is believed that the role of such mechanisms is to protect the stomach contents from shifting back into the esophagus. Several factors come together in order to fulfill this role. One contribution is the slanted position of the stomach in relation to the long axis of the esophagus. This is known as the anatomical angle of His [14]. Another contribution is the sphincter action of the diaphragmatic crura [5]. Positive pressure in the abdominal cavity functions like an additional sphincter by compressing the distal segment of the esophagus located in the abdominal cavity [15]. The distal portion of the esophagus is thought to act as a valve that protects against reflux by closing the entry of the stomach into the esophagus from the inside when intra-abdominal pressure is increased.

Although manometry shows increased pressure in the distal end of the esophagus, there is still no agreement in regards to the presence of separate muscle fibers which can be attributed to the role of the lower esophageal sphincter (LES) in antireflux mechanisms [6]. The pressure build up in the gastric fundus helps to create a mucosal flap valve which presses against the lower esophagus and thus blocks the gastro-esophageal passage $[7,16]$. Oblique muscles, also referred to as "sling fibers", are arranged in a C-shaped manner below the LES and are thought to be responsible for the creation of the flap valve mechanism $[6,7]$. Recently, a thickened circular muscular layer in the distal esophagus was described, suggesting the presence of a lower internal esophageal sphincter. However, this matter merits further study [17].

The exact cause of esophageal hiatal hernias has yet to be elucidated but several theories have been put forth. A multigenerational family study has suggested that hiatal hernias are hereditary and transferred by autosomal dominant inheritance [18]. Furthermore, body build and exertion also seem to play a role in hernia development. Excessive body weight has been associated with hiatal hernia development, such as that seen in obese individuals [19]. Obesity is strongly correlated to increased intra-gastric pressure and it is believed that this leads to esophagogastric junction impairment resulting in the development of hiatal hernias [19]. Similarly, among power lifting athletes, it is proposed that hiatal hernias are a result of supraphysiological increases in intra-abdominal pressure during weight lifting [20].

Mittal and Kassab [21] suggest that the initial cause of the development of hiatal hernias is sporadic incidents of GERD that cause inflammatory reactions in the esophagus. These in turn shorten the esophagus due to, among other things, longitudinal muscle contraction. The latter becomes a cause of intussusceptions of the stomach into the esophageal hiatus which broadens the hiatus size and exacerbates symptoms of GERD and inflammation. In such a manner, a feedback of self-escalating mechanisms that favor hiatal dilatation and hernia formation is entered. According to Dodds et al. [22] it is the transient lower esophageal sphincter relaxations (TLOSR), which normally allow belching to occur, that are responsible for the initiation of reflux events and esophagitis.

The initial expansion of the esophageal hiatus seems to be a prelude to further events leading to the formation of a hiatal hernia and acid reflux. Loosening of the hiatus makes it possible to reverse both the esophagus and the stomach cardia into the chest, in case of favorable circumstances such as air belching, vomiting, weight lifting, and other sorts of extreme increases in abdominal pressure. Wedging the cardia in the hiatus will further expand it and simultaneously broaden the angle of His, which creates a risk for hernia development. Hiatal width alone is not the only attribute of hiatal hernia origin. A very important role is also played by the phrenico-esophageal fascia. In fact, there exist two fascias: an upper and lower one. The bottom fascia can be further subdivided into upper and lower laminas. In young people, that fascia attaches the esophagus to the diaphragm flexibly but tightly. It allows for some shifts and movements of the esophagus under the influence of different forces which are present due to jumping, coughing, vomiting, and even swallowing [23, 24]. As the hiatus undergoes actions of these forces over the course of several years, connections with the esophagus may be loosened. Due to the mobility of the esophagus and esophagogastric junction, the 
fascia can be further damaged, giving rise to the formation of sliding hiatal hernias.

\section{Hernia with Gastric Fold \\ Prolapsed - Is it a Form of Sliding Hernia or a Completely Different Entity?}

Although the relationship of gastric fold prolapses to GERD and hiatal hernias remains unclear, there is some evidence to warrant the consideration of hernia with gastric fold prolapses as a distinct phenomenon. Gastroscopic examinations of patients presenting with GERD symptoms or epigastric pain have revealed prolapses of gastric mucosa into the esophagus particularly during retching $[25,26]$. A retrospective study of 516 GERD patients revealed the presence of such a prolapse in $13.5 \%$ of patients [25]. Most notably, only $29 \%$ of patients with a prolapse had a sliding hiatal hernia, indicating that the presence or absence of the hernia did not influence the clinical presentation [25]. It is proposed that retching causes gastric prolapse and subsequent mucosal injury [26]. The most prominent endoscopic features of this condition include a swollen and bleeding fold of mucosa prolapsing into the esophagus [26]. Thus, careful endoscopic examinations should be performed to take note of possible protrusions in order to distinguish such a presentation (Fig. 2) from the typical course of hiatal hernia as depicted in Fig. 3.

A thorough assessment of patients with gastric prolapse revealed that some of the presenting symptoms differ from those typically associated with GERD. Epigastric and retrosternal pain with the need to belch to decrease or resolve pain were more frequently reported by patients with gastric prolapse [25]. Dysphagia and reflux symptoms

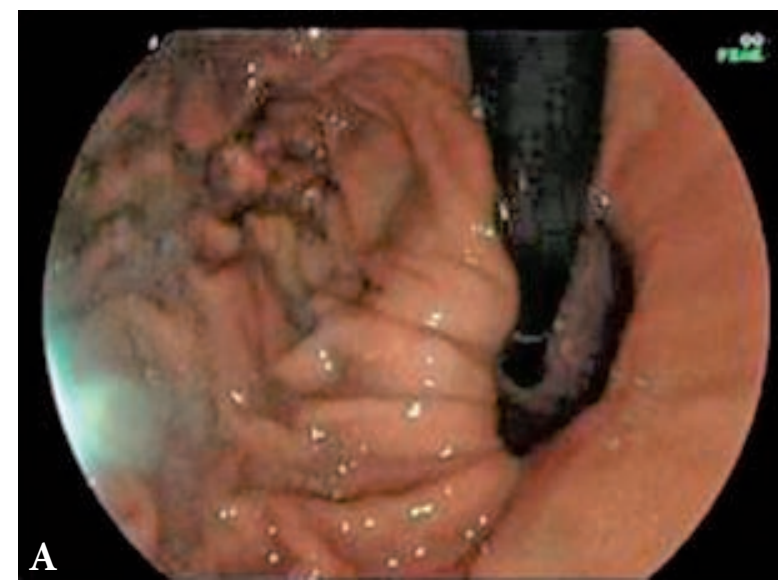

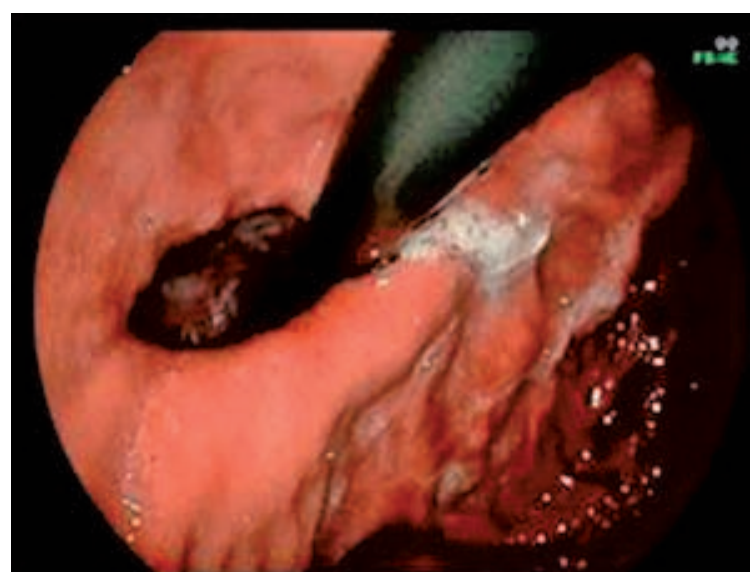

Fig. 3. Hiatal hernia endoscopy (photo made by Andrzej Gryglewski)

were also more common [25]. In comparison to GERD symptoms, pain experienced in patients with a gastric prolapse was accompanied by a radiating burning sensation running from the stomach to the throat [25]. Some studies reported a decrease in the occurrence or intensity of esophagitis, suggesting that the protruded gastric mucosa protects the esophagus from excess acid reflex $[25,26]$. However the stringency of the latter findings remains to be further scrutinized.

Myllarniemi and Saario [26] recommend treating gastric prolapse as a disease separate from GERD and hiatal hernias. According to the authors, chest pain and dental erosions caused by acid reflux during the night were distinguishing symptoms typical of that kind of hernia. The authors suggest that the cause of such a condition is the dysfunction of the His angle mechanism. According to that conception, the enlargement of hiatal diameter decreases the efficiency of the valve, causing it to protrude into the esophagus, leading to discomfort.

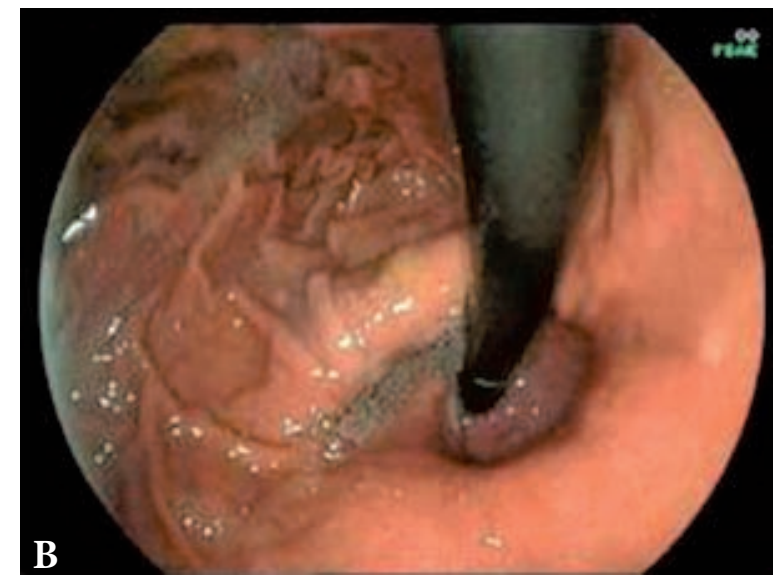

Fig. 2. A. Protruded mucosal fold in a hiatal hernia. B. Backing up of the mucosal fold (photo made by Andrzej Gryglewski) 
Despite these differences, hernias with gastric prolapse appear to present a variation of the pathological mechanisms associated with sliding hiatal hernias, notably enlargement of the hiatus and esophageal valve dysfunction. The mobility of the gastric mucosal fold is the result of the presence of smooth muscle in the muscularis mucosae layer. Under normal physiological conditions, the gastric mucosal fold and the angle of His create a valve that functions as a protective antireflux mechanism, preventing gastric enzymes from entering the esophagus. However, in some situations the valve itself may protrude into the esophagus. Symptoms of such a condition are primarily caused by mechanical trauma resulting from the fold being constricted in the esophagus and to a lesser degree by the acid reflux. Although sliding hiatal hernia with gastric prolapse sometimes shares the commonality of wedging itself in the esophageal hiatus, the gastric cardia is rather less mobile and seldom wedges in the esophageal hiatus mainly because of the presence of intact esophageal fascia and ligaments retaining the cardia in its place. Under the influence of increased abdominal pressure, the widened esophageal hiatus can promote the gastric mucosa to slide into the esophagogastric junction and cause symptoms $[6,15,25,26]$.

\section{Further Questions Regarding Hiatal Hernia Development}

It is unclear whether hernias develop from external environmental factors, such as training injuries, or if they reflect systemic metabolic disorders. Some studies propose that variation in tissue composition, in the case of primary and recurrent incisional hernias, may play a role in hernia development. For example, some patients reveal alternations in the composition of collagen I and III fibers [27]. A decrease in the collagen I: III ratio has been associated with recurrent hernia formation [27]. Equally, some investigations report a depletion of elastic fibers in ligaments supporting the gastro-esophageal junction in relation to the condition [28]. Furthermore, transmission electron microscopy has revealed ultra-structural abnormalities in the muscular tissue of the crura in patients with hiatal hernias [29]. It remains unclear if these findings represent a primary alteration or are the byproducts of hernia formation. The etiology of hiatal hernia development remains to be further elucidated.

\section{Conclusions}

The formation of different types of hiatal hernias and the development of GERD stem primarily from changes in the anatomy of the esophagogastric junction which compromises the normal antireflux mechanisms. Pathology is primarily caused by the loosening of the fibers of the phrenoesophageal membrane, which causes the esophagus to recede from the spine. As a result, the crura of the diaphragm elongate and promote the permanent herniation of the stomach cardia into the esophageal hiatus. However, even when the stomach fascia is intact, loose folds of gastric mucosa may prolapse into the hiatus and the patient will present with a different array of symptoms. In such instances, it becomes unclear whether this type of hernia should be considered as an independent ailment or merely as a part of the spectrum of conditions associated with esophageal hiatal hernias. Such a distinction is complicated by the multifactorial nature of the etiology of the disease, which involves the interplay of genetic and environmental factors.

The exact point at which hernia development begins has yet to be understood and may shed light on both the etiology and appropriate therapeutic interventions required. Identifying the origin of the pathology may shift the focus of treatment from management to one of prevention.

\section{References}

[1] Loffeld RJ, Liberov B, Dekkers PE: The changing prevalence of upper gastrointestinal endoscopic diagnoses: a single-centre study. Neth J Med 2012, 70, 222-226.

[2] Gordon C, Kang JY, Neild PJ, Maxwell JD: The role of the hiatus hernia in gastro-oesophageal reflux disease. Aliment Pharmacol Ther 2004, 20, 719-732.

[3] Dean C, Etienne D, Carpentier B, Gielecki J, Tubbs RS, Loukas M: Hiatal hernias. Surg Radiol Anat 2012, 34, $291-299$.

[4] Loukas M, Wartmann ChT, Tubbs RS, Apaydin N, Louis RG Jr, Gupta AA, Jordan R: Morphologic variation of the diaphragmatic crura: a correlation with pathologic processes of the esophageal hiatus? Folia Morphol 2008, 67, 273-279.

[5] Mittal RK, Rochester DF, McCallum RW: Effect of the diaphragmatic contraction on lower oesophageal sphincter pressure in man. Gut 1987, 28, 1564-1568.

[6] Mittal RK, Balaban DH: The esophagogastric junction. N Engl J Med 1997, 336, 924-932.

[7] Kaneyama H, Kaise M, Arakawa H, Arai Y, Kanazawa K, Tajiri H: Gastroesophageal flap valve status distinguishes clinical phenotypes of large hiatal hernia. World J Gastroenterol 2010, 16, 6010-6015. 
[8] Kahrilas PJ, Kim HC, Pandolfino JE: Approaches to the diagnosis and grading of hiatal hernia. Best Pract Res Clin Gastroenterol 2008, 22, 601-616.

[9] Shafik A, Shafik A, El-Sibai O, Shafik I: Physioanatomic study of the diaphragmatic crura: the identification of autonomous "gastroesophageal sphincter". J Invest Surg 2005, 18, 135-142.

[10] Fitzgibbons RJ, Greenburg AG: Nyhus \& Condon’s Hernia. Lippincott Williams \& Wilkins, Philadelphia 2001, $5^{\text {th }}$ ed., 479-514.

[11] Skandalakis J, Colborn G, Weidman T, et al.: Diaphragm. In: Skandalakis' Surgical Anatomy. Eds.: Skandalakis JE, Colburn GL, Weidman TA, Foster, Jr. RS, Kingsworth AN, Skandalakis LJ, Skandalakis PN, Mirilas PS, McGrawHill, New York 2004.

[12] Awais O, Luketich JD: Management of giant paraesophageal hernia. Minerva Chir 2009, 64, 159-168.

[13] Paterson WG: The normal antireflux mechanism. Chest Surg Clin N Am 2001, 11, 473-483.

[14] Fujiwara Y, Nakagawa K, Kusunoki M, Tanaka T, Yamamura T, Utsunomiya J: Gastroesophageal reflux after distal gastrectomy: possible significance of the angle of His. Am J Gastroenterol 1998, 93, 11-15.

[15] Atkinson M: Mechanisms protecting against gastro-esophageal reflux: a review. Gut 1962, 3, 1-15.

[16] Hill L, Kozarek RA, Kraemer SJ, Aye RW, Mercer CD, Low DE, Pope CE 2nd: The gastroesophageal flap valve: in vitro and in vivo observations. Gastrointest Endosc 1996, 44, 541-547.

[17] Apaydin N, Uz A, Elhan A, Loukas M, Tubbs RS: Does an anatomical sphincter exist in the distal esophagus? Surg Radiol Anat 2008, 30, 11-16.

[18] Carré IJ, Johnston BT, Thomas PS, Morrison PJ: Familial hiatal hernia in large five generation family confirming true autosomal dominant inheritance. Gut 1999, 45, 649-652.

[19] Pandolfino JE, El-Serag HB, Zhang Q, Shah N, Ghosh SK, Kahrilas PJ: Obesity: a challenge to esophagogastric junction integrity. Gastroenterology 2006, 130, 639-649.

[20] Smith AB, Dickerman RD, McGuire CS, East JW, McConathy WJ, Pearson HF: Pressure-overload-induced sliding hiatal hernia in power athletes. J Clin Gastroenterol 1999, 28, 352-354.

[21] Mittal RK, Kassab GS: Esophagogastric junction opening: does it explain the difference between normal subjects and patients with reflux disease? Gastroenterology 2003, 125, 1258-1260.

[22] Dodds WJ, Dent J, Hogan WJ, Helm JF, Hauser R, Patel GK, Egide MS: Mechanisms of gastro-oesophageal reflux in patients with reflux esophagitis. N Engl J Med 1982, 307, 1547-1552.

[23] Eliska O: Phreno-oesophageal membrane and its role in the development of hiatal hernia. Acta Anat (Basel) 1973, $86,137-150$.

[24] Sugarbaker DJ, Rattan S, Goyal R: Swallowing induces sequential activation of esophageal longitudinal smooth muscle. Am J Physiol 1984, 247, 15-19.

[25] Aramini B, Mattioli S, Lugaresi M. Brusori S, Di Simone MP, D’Ovidio F: Prevalence and clinical picture of gastroesophageal prolapse in gastroesophageal reflux disease. Dis Esophagus 2012, 25, 491-497.

[26] Myllärniemi H, Saario I: A new type of slipping hiatus hernia. Ann Surg 1985, 202, 159-161.

[27] Brown SR, Melman L, Jenkins E, Deeken C, Frisella MM, Brunt LM, Eagon JC, Matthews BD: Collagen type I: III ratio of the gastroesophageal junction in patients with paraesophageal hernias. Surg Endosc 2011, 25, 13901394.

[28] Curci JA, Melman LM, Thompson RW, Soper NJ, Matthews BD: Elastic fiber depletion in the supporting ligaments of the gastroesophageal junction: a structural basis for the development of hiatal hernia. J Am Coll Surg 2008, 2, 191-196.

[29] Fei L, del Genio G, Rossetti G, Sampaolo S, Moccia F, Trapani V, Cimmino M, del Genio A: Hiatal hernia recurrence: surgical complication or disease? Electron microscope findings of the diaphragmatic pillars. J Gastrointest Surg 2009, 13, 459-464.

\section{Address for correspondence:}

Krzysztof A. Tomaszewski

Department of Anatomy

Jagiellonian University Medical College

Kopernika 12

31-034 Kraków

Poland

Tel.: +48 124229511

E-mail: krtomaszewski@gmail.com

Conflict of interest: None declared

Received: 11.06.2013

Revised: 14.11.2013

Accepted: 23.07.2014 